\title{
ON THE EXISTENCE OF A GLOBAL NEIGHBOURHOOD
}

\author{
TOM COATES AND HIROSHI IRITANI
}

\begin{abstract}
Suppose that a complex manifold $M$ is locally embedded into a higher-dimensional neighbourhood as a submanifold. We show that, if the local neighbourhood germs are compatible in a suitable sense, then they glue together to give a global neighbourhood of $M$. As an application, we prove a global version of Hertling-Manin's unfolding theorem for germs of TEP structures; this has applications in the study of quantum cohomology.
\end{abstract}

\section{INTRODUCTION}

We prove:

Theorem 1. Let $M$ be a complex manifold of dimension $m$ and let $\mathcal{A}$ be a sheaf of $\mathbb{C}$-algebras over $M$. Suppose that there exist a natural number $n$ and a morphism of sheaves of $\mathbb{C}$-algebras $\pi: \mathcal{A} \rightarrow$ $\mathcal{O}_{M}$ such that for each $x \in M$ there exists an open neighbourhood $U$ of $x$ and an isomorphism $\left.\mathcal{A}\right|_{U} \cong \iota^{-1} \mathcal{O}_{U \times \mathbb{C}^{n}}$, where $\iota: U \hookrightarrow U \times \mathbb{C}^{n}$ is the embedding $x \mapsto(x, 0)$, such that the following diagram commutes:

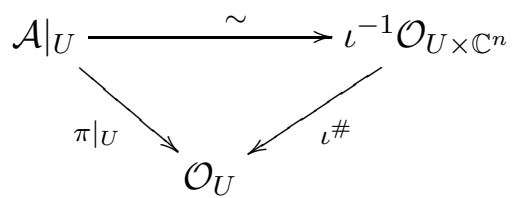

Here $\iota^{\#}$ is the canonical morphism induced by $\iota$. Then:

(i) There exist a complex manifold $M^{\prime}$ of dimension $m+n$ and a closed embedding $\iota: M \rightarrow M^{\prime}$ such that we have an isomorphism $\mathcal{A} \cong \iota^{-1} \mathcal{O}_{M^{\prime}}$ of sheaves of $\mathbb{C}$-algebras which commutes with the surjections to $\mathcal{O}_{M}$.

(ii) The manifold-germ $M^{\prime}$ is unique up to unique isomorphism in the following sense. If we have two complex manifolds $M_{1}^{\prime}, M_{2}^{\prime}$, two closed embeddings $\iota_{1}: M \rightarrow M_{1}^{\prime}, \iota_{2}: M \rightarrow M_{2}^{\prime}$, and an isomorphism of sheaves of $\mathbb{C}$-algebras $\phi: \iota_{1}^{-1} \mathcal{O}_{M_{1}^{\prime}} \rightarrow \iota_{2}^{-1} \mathcal{O}_{M_{2}^{\prime}}$ commuting with the surjections to $\mathcal{O}_{M}$, then $\phi$ is induced by a biholomorphic map $\varphi: N_{2} \rightarrow N_{1}$ between open neighbourhoods $N_{i}$ of $M$ in $M_{i}^{\prime}, i \in\{1,2\}$, such that $\varphi$ is the identity map on $M$. Such a map $\varphi$ is unique as a germ of maps on a neighbourhood of $M$.

We also discuss the extension of sheaves, proving:

Theorem 2. Suppose that $\iota: M \hookrightarrow M^{\prime}$ is a closed embedding of complex manifolds. Let $\mathcal{A}$ be the sheaf of $\mathbb{C}$-algebras $\mathcal{A}=\iota^{-1} \mathcal{O}_{M^{\prime}}$ over $M$ and let $\mathcal{B}$ be a coherent $\mathcal{A}$-module. Then:

(i) There exist an open neighbourhood $N$ of $M$ in $M^{\prime}$ and a coherent $\mathcal{O}_{N}$-module $\mathcal{B}^{\prime}$ on $N$ such that $\iota^{-1} \mathcal{B}^{\prime} \cong \mathcal{B}$ as sheaves of $\mathcal{A}$-modules.

(ii) The sheaf-germ $\mathcal{B}^{\prime}$ is unique up to unique isomorphism in the following sense. If we have two coherent $\mathcal{O}_{N}$-modules $\mathcal{B}_{1}^{\prime}$ and $\mathcal{B}_{2}^{\prime}$ on a neighbourhood $N$ of $M$ in $M^{\prime}$ and isomorphisms $\iota^{-1} \mathcal{B}_{1}^{\prime} \cong \mathcal{B} \cong \iota^{-1} \mathcal{B}_{2}^{\prime}$ of $\mathcal{A}$-modules, with $\phi: \iota^{-1} \mathcal{B}_{1}^{\prime} \rightarrow \iota^{-1} \mathcal{B}_{2}^{\prime}$ denoting the composite isomorphism, then $\phi$ is induced by an isomorphism $\Phi:\left.\left.\mathcal{B}_{1}^{\prime}\right|_{P} \rightarrow \mathcal{B}_{2}^{\prime}\right|_{P}$ of $\mathcal{O}_{P}$-modules on an open

Key words and phrases. complex manifold, real analytic manifold, neighbourhood, germ, gluing, Hausdorff, complexification, unfolding, Frobenius manifold, TEP structure. 
neighbourhood $P$ of $M$ in $N$. Such a morphism $\Phi$ is unique as a germ of homomorphisms over a neighbourhood of $M$.

Remark 3. By Oka's coherence theorem, $\mathcal{O}_{M^{\prime}}$ is coherent and hence $\mathcal{A}=\iota^{-1} \mathcal{O}_{M^{\prime}}$ is also coherent as a sheaf of algebras. Therefore being a coherent $\mathcal{A}$-module is equivalent to being locally finitely presented as an $\mathcal{A}$-module, i.e. for each $x \in M$, there exists an open neighbourhood $U$ of $x$ in $M$ and an exact sequence of $\mathcal{A}$-modules:

$$
\left.\mathcal{A}_{U}^{\oplus k} \longrightarrow \mathcal{A}_{U}^{\oplus l} \longrightarrow \mathcal{B}\right|_{U} \longrightarrow 0
$$

for some $k, l \in \mathbb{N}$. See e.g. [9, Appendix].

Remark 4. If in addition $\mathcal{B}$ is locally free as an $\mathcal{A}$-module in Theorem 2 , then $\mathcal{B}^{\prime}$ becomes locally free as an $\mathcal{O}_{N}$-module in a neighbourhood $N$ of $M$, because the stalk $\mathcal{B}_{x}^{\prime}$ at each $x \in M$ is a free $\mathcal{O}_{M^{\prime}, x^{-}}$module.

Remark 5. We have stated Theorems 1 and 2 in the category of holomorphic manifolds, but the same statements hold true, with the same proofs, in the real analytic category.

We can reformulate our results as an equivalence of categories. Namely, for Theorem 1, the category of sheaves $\mathcal{A}$ of $\mathbb{C}$-algebras on $M$ equipped with surjections $\pi: \mathcal{A} \rightarrow \mathcal{O}_{M}$ satisfying the local condition (1) is equivalent to the category of germs of neighbourhoods $\iota: M \hookrightarrow M^{\prime}$ of $M$. For Theorem 2 , the category of coherent $\mathcal{A}$-modules is equivalent to the category of germs of coherent sheaves on a neighbourhood of $M$ in $M^{\prime}$. It is not difficult to modify the discussion below to prove these categorical equivalences.

In the real analytic category (Remark 5), Theorem 1 may be viewed as a generalization of the existence theorem for the complexification of a real-analytic manifold, see e.g. [12]. In the $C^{\infty}$ category, Lemma 8 below is not valid (see e.g. [11]) and our results do not hold. However, most of the arguments for Theorem 1 work if we can take representatives of neighbourhood germs and $C^{\infty}$ gluing maps between them which satisfy cocycle conditions as germs; similar arguments appear in the context of Kuranishi structures [5,8]. Our original motivation was to globalize the unfolding of Frobenius-type structures (or meromorphic connections, or TEP structures) which has been studied by Hertling-Manin [7] and Reichelt [10] on the level of germs. We give a global version of Hertling-Manin's unfolding theorem for TEP structures in section 4 below.

Notation 6. We require that manifolds be paracompact.

Notation 7. We write $A \Subset B$ if and only if $A$ is a relatively compact subset of $B$.

Acknowledgments. T.C. thanks Eugenia Cheng for useful correspondence. H.I. thanks Ono Kaoru and Ken-ichi Yoshikawa for useful discussions.

\section{The Proof of Theorem 1}

Our assumptions on $\mathcal{A}$ imply that we can find a locally-finite open covering $\left\{W_{i}: i \in I\right\}$ of $M$ with index set $I$ such that $\left.\mathcal{A}\right|_{W_{i}} \cong \iota^{-1} \mathcal{O}_{W_{i} \times Z_{i}}$, where $Z_{i}$ is a copy of $\mathbb{C}^{n}$ and $\iota: W_{i} \rightarrow W_{i} \times Z_{i}$ is the embedding $x \mapsto(x, 0)$. Without loss of generality we can assume both that $W_{i}$ is a co-ordinate neighbourhood on $M$ (i.e. is identified with an open subset of $\mathbb{C}^{m}$ ) and that $W_{i}$ is relatively compact in $M$. We take locally finite coverings $\left\{U_{i}: i \in I\right\},\left\{V_{i}: i \in I\right\}$ with the same index set $I$ such that $U_{i} \Subset V_{i} \Subset W_{i}$. We write $W_{i j}:=W_{i} \cap W_{j}, V_{i j}:=V_{i} \cap V_{j}, V_{i j k}:=V_{i} \cap V_{j} \cap V_{k}$. The basic fact we use for the gluing is the following Lemma.

Lemma 8. Let $U \subset \mathbb{C}^{m}$ be an open set and let $\iota: U \rightarrow U \times \mathbb{C}^{n}$ be the embedding $x \mapsto(x, 0)$. Let $\phi: \iota^{-1} \mathcal{O}_{U \times \mathbb{C}^{n}} \rightarrow \iota^{-1} \mathcal{O}_{U \times \mathbb{C}^{n}}$ be a homomorphism of sheaves of $\mathbb{C}$-algebras which commutes with the natural surjections to $\mathcal{O}_{U}$. Then: 
(a) there exists an open neighbourhood $U^{\prime}$ of $U \times\{0\}$ in $U \times \mathbb{C}^{n}$ and a holomorphic map $\varphi: U^{\prime} \rightarrow$ $U \times \mathbb{C}^{n}$ which is the identity on $U \times\{0\}$ such that $\phi$ coincides with the pull-back by $\varphi$;

(b) if $\phi$ is an isomorphism then the map $\varphi$ is a biholomorphic isomorphism onto its image.

Proof of Lemma 8. Statement (a) implies statement (b), by the inverse function theorem, so we prove (a). Consider first the case where $m=0$ and $U$ is a point. Then $\phi$ is a $\mathbb{C}$-algebra endomorphism of the ring $\iota^{-1} \mathcal{O}_{\mathbb{C}^{n}}=\mathbb{C}\left\{z_{1}, \ldots, z_{n}\right\}$ of convergent power series which preserves the maximal ideal $\mathfrak{m}=\left(z_{1}, \ldots, z_{n}\right)$. Because such a $\phi$ is continuous with respect to the $\mathfrak{m}$-adic topology, $\phi$ is determined by the images of the generators $z_{1}, \ldots, z_{n}$. The images determine a holomorphic map $\varphi:\left(z_{1}, \ldots, z_{n}\right) \mapsto\left(\phi\left(z_{1}\right), \ldots, \phi\left(z_{n}\right)\right)$ which is defined on a neighbourhood $U^{\prime}$ of 0 in $\mathbb{C}^{n}$, and $\phi$ coincides with the pull-back by $\varphi$.

Consider now the general case. Let $t_{1}, \ldots, t_{m}$ denote the standard co-ordinates on $U \subset \mathbb{C}^{m}$ and let $z_{1}, \ldots, z_{n}$ denote the standard co-ordinates on $\mathbb{C}^{n}$. Then the images of $t_{1}, \ldots, t_{m}$ and $z_{1}, \ldots, z_{n}$ under $\phi$ give global sections of $\iota^{-1} \mathcal{O}_{U \times \mathbb{C}^{m}}$, and thus they define a holomorphic map

$$
\varphi:\left(t_{1}, \ldots, t_{m}, z_{1}, \ldots, z_{n}\right) \mapsto\left(\phi\left(t_{1}\right), \ldots, \phi\left(t_{m}\right), \phi\left(z_{1}\right), \ldots, \phi\left(z_{n}\right)\right)
$$

on a neighbourhood $U^{\prime}$ of $U \times\{0\}$ in $U \times \mathbb{C}^{n}$. Since $\phi$ commutes with the surjections to $\mathcal{O}_{U}$, $\varphi$ restricts to the identity map on $U \times\{0\}$. The pull-back $\varphi^{\star}: \iota^{-1} \mathcal{O}_{U \times \mathbb{C}^{n}} \rightarrow \iota^{-1} \mathcal{O}_{U \times \mathbb{C}^{n}}$ defines a homomorphism of sheaves of $\mathbb{C}$-algebras commuting with the surjections to $\mathcal{O}_{U}$. The $m=0$, $U=$ point case implies that $\left(\varphi^{\star}\right)_{t}=\phi_{t}$ on the stalk at every point $t \in U$. Thus $\varphi^{\star}=\phi$.

The composite isomorphism $\left.\iota^{-1} \mathcal{O}_{W_{i j} \times Z_{i}} \cong \mathcal{A}\right|_{W_{i j}} \cong \iota^{-1} \mathcal{O}_{W_{i j} \times Z_{j}}$ induces a biholomorphic isomorphism $\varphi_{i j}: N_{i j} \rightarrow N_{j i}$ for each $i, j \in I$, where $N_{i j}$ is an open neighbourhood of $W_{i j} \times\{0\}$ in $W_{i j} \times Z_{i}$ and $\varphi_{i j}$ is the identity on $W_{i j} \times\{0\}$. Note that $N_{i j}$ and $N_{j i}$ are subsets of different spaces. Without loss of generality we may assume that $N_{i i}=W_{i} \times Z_{i}$ and that $\varphi_{i i}$ is the identity map. Define:

$$
\begin{aligned}
& O_{i}:=V_{i} \times Z_{i} \\
& O_{i j}:=\left(V_{i j} \times Z_{i}\right) \cap N_{i j} \cap \varphi_{i j}^{-1}\left(V_{i j} \times Z_{j}\right)
\end{aligned}
$$

Then $O_{i j}$ is an open subset of $O_{i}$ which contains $V_{i j} \times\{0\}$. By restricting $\varphi_{i j}$ to $O_{i j}$, we obtain a biholomorphic isomorphism $\varphi_{i j}: O_{i j} \rightarrow O_{j i}$ such that $\left.\varphi_{i j}\right|_{V_{i j} \times\{0\}}$ is the identity map.

Lemma 9. There exist open subsets $Q_{i}$ of $O_{i}, i \in I$, such that for each $i, j \in I$ we have:

(a) $Q_{i} \Subset O_{i}$;

(b) $U_{i} \times\{0\} \subset Q_{i} \subset U_{i} \times Z_{i}$;

(c) $Q_{i j} \Subset O_{i j}$, where $Q_{i j}:=Q_{i} \cap O_{i j} \cap \varphi_{i j}^{-1}\left(Q_{j}\right)$.

Proof of Lemma 9. Denote by $\bar{U}_{i}$ the closure of $U_{i}$ in $V_{i}$; by assumption $\bar{U}_{i}$ is compact. We have that $\bar{U}_{i} \cap \bar{U}_{j}$ is contained in $V_{i j}$, and hence that $\left(\bar{U}_{i} \cap \bar{U}_{j}\right) \times\{0\} \subset O_{i j}$. Fix $i, j \in I$, and fix a relatively compact open subset $P$ of $O_{i j}$ such that $P$ contains $\left(\bar{U}_{i} \cap \bar{U}_{j}\right) \times\{0\}$; such a subset exists because the set $\bar{U}_{i} \cap \bar{U}_{j}$ is compact. Set:

$$
Q_{i}(n):=U_{i} \times\left\{x \in Z_{i}:|x|<\frac{1}{n}\right\}
$$

noting that $Q_{i}(n)$ satisfies conditions (a) and (b) of the Lemma.

We claim that there exists $n$ such that

$$
Q_{i}(n) \cap O_{i j} \cap \varphi_{i j}^{-1}\left(Q_{j}(n)\right) \subset P
$$

Suppose, on the contrary, that for each $n$ there exists an element $x_{n} \in Q_{i}(n) \cap O_{i j} \cap \varphi_{i j}^{-1}\left(Q_{j}(n)\right)$ such that $x_{n} \notin P$. After passing to a subsequence, we have that $\left(x_{n}\right)$ converges to a limit $x \in \bar{U}_{i} \times\{0\}$. Thus $\left(x_{n}\right)$ converges in $O_{i}$. On the other hand each $x_{n}$ lies in the closed subset $O_{i} \backslash P$ of $O_{i}$, and so $x \in O_{i} \backslash P$. Thus $x$ lies in $\left(\bar{U}_{i} \backslash \bar{U}_{j}\right) \times\{0\}$. Now $x_{n}$ lies in $O_{i j}$ for each $n$, hence $x_{n}$ lies in $V_{i j} \times Z_{i}$ 
and the limit $x$ lies in the closure of $V_{i j}$ in $M$. However the closure of $V_{i j}$ is contained in $W_{i j}$. Recall that $\varphi_{i j}$ is defined and continuous on the open neighbourhood $N_{i j}$ of $W_{i j} \times\{0\}$ in $W_{i}^{\prime}$. Thus:

$$
\varphi_{i j}(x)=\lim _{n \rightarrow \infty} \varphi_{i j}\left(x_{n}\right)
$$

The right hand side here converges to an element in $\bar{U}_{j} \times\{0\}$, since $\varphi_{i j}\left(x_{n}\right) \in Q_{j}(n)$. This is a contradiction: we have shown that $x \in \bar{U}_{i} \backslash \bar{U}_{j}$, and $\left.\varphi_{i j}\right|_{W_{i j} \times\{0\}}$ is the identity map. Thus, for each $i$ and $j \in I$, there exists an integer $n=n(i, j)$ such that (3) holds.

Since $V_{i}$ is relatively compact in $M$ and since the covering $\left\{V_{i}: i \in I\right\}$ is locally finite, only finitely many $V_{j}$ have non-empty intersection with a fixed $V_{i}$. Thus we can define:

$$
\begin{aligned}
& n(i):=\max \left\{n(i, j): j \in I \text { such that } V_{i} \cap V_{j} \neq \varnothing\right\} \\
& Q_{i}:=Q_{i}(n(i))
\end{aligned}
$$

to obtain open sets $\left\{Q_{i}: i \in I\right\}$ with the properties claimed.

Lemma 10. Let $\left\{Q_{i}: i \in I\right\}$ be such that $Q_{i}$ is an open subset of $O_{i}$ and that properties (a-c) in Lemma 9 hold. Then the image of the map $Q_{i j} \rightarrow Q_{i} \times Q_{j}$ given by (inclusion, $\left.\varphi_{i j}\right)$ is closed in $Q_{i} \times Q_{j}$.

Proof of Lemma 10. Let $\left(x_{n}\right)$ be a sequence in $Q_{i j}$ such that $\left(x_{n}\right)$ converges in $Q_{i}$ and $\left(\varphi_{i j}\left(x_{n}\right)\right)$ converges in $Q_{j}$. Let $x$ denote the limit of $\left(x_{n}\right)$ in $Q_{i}$. Since $Q_{i j}$ is relatively compact in $O_{i j}$, the limit $x$ lies in $O_{i j}$. But $\left(\varphi_{i j}\left(x_{n}\right)\right)$ converges in $Q_{j}$ and thus $\varphi_{i j}(x) \in Q_{j}$, or in other words $x \in \varphi_{i j}^{-1}\left(Q_{j}\right)$. Thus $x \in Q_{i j}$.

Lemma 11. There exist open subsets $Q_{i}$ of $O_{i}, i \in I$, such that properties ( $\left.a-c\right)$ in Lemma 9 hold and further, for each $i, j, k \in I$, we have:

(d) $Q_{i j} \cap Q_{i k} \subset \varphi_{i j}^{-1}\left(O_{j k}\right)$;

(e) $\varphi_{j k} \circ \varphi_{i j}=\varphi_{i k}$ on $Q_{i j} \cap Q_{i k}$.

Note that condition (d) guarantees that the composition in (e) is well-defined.

Proof of Lemma 11. Let $\left\{Q_{i}: i \in I\right\}$ be such that, for each $i \in I, Q_{i}$ is an open subset of $O_{i}$ and that properties $(\mathrm{a}-\mathrm{c})$ hold. Such subsets exist by Lemma 9. Define:

$$
Q_{i j k}:=Q_{i j} \cap Q_{i k}
$$

Let $\bar{Q}_{i j}$ denote the closure of $Q_{i j}$ in $O_{i j}$. This is compact, and hence $\bar{Q}_{i j}$ is at the same time the closure of $Q_{i j}$ in $O_{i}$. Let $\bar{Q}_{i j k}$ denote the closure of $Q_{i j k}$ in $O_{i}$. This is contained in the compact set $\bar{Q}_{i j} \cap \bar{Q}_{i k}$, hence in particular is contained in $O_{i j} \cap O_{i k}$.

We claim that there exists an open neighbourhood $N_{i j k}$ of $\bar{Q}_{i j k} \cap\left(U_{i} \times\{0\}\right)$ in $O_{i j} \cap O_{i k}$ such that:

- $N_{i j k} \subset \varphi_{i j}^{-1}\left(O_{j k}\right)$; and

- $\varphi_{j k} \circ \varphi_{i j}=\varphi_{i k}$ holds on $N_{i j k}$.

It suffices to show that each $x$ in $\bar{Q}_{i j k} \cap\left(U_{i} \times\{0\}\right)$ has an open neighbourhood $N_{x}$ in $O_{i j} \cap O_{i k}$ such that $N_{x} \subset \varphi_{i j}^{-1}\left(O_{j k}\right)$ and that $\varphi_{j k} \circ \varphi_{i j}=\varphi_{i k}$ holds on $N_{x}$. Let $x \in \bar{Q}_{i j k} \cap\left(U_{i} \times\{0\}\right)$. Then $x$ lies in $O_{i j} \cap O_{i k} \cap\left(U_{i} \times\{0\}\right)=\left(V_{i j k} \cap U_{i}\right) \times\{0\}$ and hence lies in $O_{i j} \cap O_{i k} \cap \varphi_{i j}^{-1}\left(O_{j k}\right)$. But $\varphi_{j k} \circ \varphi_{i j}=\varphi_{i k}$ holds as germs at $x$, and so choosing $N_{x}$ to be a sufficiently small open neighbourhood of $x$ in $O_{i j} \cap O_{i k} \cap \varphi_{i j}^{-1}\left(O_{j k}\right)$ proves the claim.

Recall that properties (a-c) in Lemma 9 hold for $\left\{Q_{i}: i \in I\right\}$ and note that, with the exception of the assertion that $U_{i} \times\{0\} \subset Q_{i}$, these properties are preserved under shrinking the sets $Q_{i}$. For a fixed $i \in I$, there are only finitely many pairs $(j, k) \in I \times I$ such that the triple intersection $V_{i j k}$ is nonempty. Thus, as $Q_{i j k} \subset V_{i j k} \times Z_{i}$, there are only finitely many pairs $(j, k) \in I \times I$ such that 
$Q_{i j k}$ is nonempty. Let $\left(j_{1}, k_{1}\right), \ldots,\left(j_{f}, k_{f}\right)$ be all such pairs. We shrink $Q_{i}$ inductively as follows: set $Q_{i}^{(0)}:=Q_{i}$, set

$$
Q_{i}^{(a)}:=\left(Q_{i}^{(a-1)} \backslash \bar{Q}_{i j_{a} k_{a}}\right) \cup\left(N_{i j_{a} k_{a}} \cap Q_{i}^{(a-1)}\right) \subset Q_{i}^{(a-1)}
$$

for $a=1, \ldots, f$, and set $Q_{i}^{\text {new }}:=Q_{i}^{(f)}$. Then $Q_{i}^{\text {new }}$ is an open subset of the original set $Q_{i}$, and it contains $U_{i} \times\{0\}$. Thus properties (a-c) in Lemma 9 hold for the new sets $\left\{Q_{i}^{\text {new }}: i \in I\right\}$. Furthermore, for each $i, j, k \in I, Q_{i j}^{\text {new }}:=Q_{i}^{\text {new }} \cap O_{i j} \cap \varphi_{i j}^{-1}\left(Q_{j}^{\text {new }}\right)$ is contained in $Q_{i j}$ and $Q_{i j k}^{\text {new }}:=$ $Q_{i j}^{\text {new }} \cap Q_{i k}^{\text {new }}$ is contained in $Q_{i j k}$. Also $Q_{i j k}^{\text {new }}$ is contained in $N_{i j k}$. Therefore properties (d) and (e) hold for $\left\{Q_{i}^{\text {new }}: i \in I\right\}$, and the Lemma is proved.

Remark. Let $Q_{i} \subset O_{i}, i \in I$, be open subsets such that properties (a-e) in Lemma 11 hold. In particular, then, $Q_{i j} \cap Q_{i k} \subset \varphi_{i j}^{-1}\left(O_{j k}\right)$. But slightly more is true: in fact $Q_{i j} \cap Q_{i k} \subset \varphi_{i j}^{-1}\left(Q_{j k}\right)$. For if $x \in Q_{i j} \cap Q_{i k}$ then $\varphi_{i j}(x) \in Q_{j i} \subset Q_{j}$ and $\varphi_{i k}(x) \in Q_{k i} \subset Q_{k}$. Also $\varphi_{j k} \circ \varphi_{i j}(x)=\varphi_{i k}(x)$, which lies in $Q_{k}$. Thus $\varphi_{i j}(x)$ lies in $Q_{j} \cap O_{j k} \cap \varphi_{j k}^{-1}\left(Q_{k}\right)=: Q_{j k}$.

We now complete the proof of Theorem 1. Choose open subsets $Q_{i} \subset O_{i}, i \in I$, such that properties (a-e) in Lemma 11 hold. (This is possible by Lemma 11.) Set $M^{\prime}$ equal to the quotient space:

$$
\left(\coprod_{i \in I} Q_{i}\right) / \sim
$$

by the equivalence relation $\sim$ generated by $x \sim \varphi_{i j}(x)$ where $x \in Q_{i j}$. We claim that $M^{\prime}$ is a complex manifold.

Let $X=\coprod_{i \in I} Q_{i}$ and consider the binary relation $R$ on $X \times X$ given by:

$$
R:=\coprod_{i, j \in I} Q_{i j} \subset \coprod_{i, j \in I} Q_{i} \times Q_{j}=X \times X
$$

where the map $Q_{i j} \rightarrow Q_{i} \times Q_{j}$ is given by (inclusion, $\varphi_{i j}$ ). Then $M^{\prime}$ is the quotient space of $X$ by the equivalence relation generated by $R$. To show that $M^{\prime}$ is a complex manifold it suffices to prove that $M^{\prime}$ is Hausdorff; hence it suffices to prove that $R$ is closed and that $R$ is an equivalence relation. We have shown that the image of the map $Q_{i j} \rightarrow Q_{i} \times Q_{j}$ is closed (Lemma 10), so $R$ is closed. It remains to show that $R$ is an equivalence relation. Reflexivity $(x \sim x)$ is obvious, since $Q_{i i}=Q_{i}$ and $\varphi_{i i}$ is the identity map. Symmetricity $(x \sim y \Longrightarrow y \sim x)$ is also obvious, since $\varphi_{i j}$ and $\varphi_{j i}$ are inverse to each other. For transitivity $(x \sim y \wedge y \sim z \Longrightarrow x \sim z)$ assume that $x \in Q_{j}$, $y \in Q_{i}, z \in Q_{k}, x \sim y$, and $y \sim z$. Then $y \in Q_{i j}$ (since $y \sim x$ ) and $y \in Q_{i k}$ (since $y \sim z$ ), thus $y \in Q_{i j} \cap Q_{i k}$. The Remark after Lemma 11 implies that $y \in \varphi_{i j}^{-1}\left(Q_{j k}\right)$, and Lemma 11 implies that $\varphi_{j k} \circ \varphi_{i j}(y)=\varphi_{i k}(y)$. But $x=\varphi_{i j}(y)$ and $z=\varphi_{i k}(y)$, so $z=\varphi_{j k}(x)$. Thus $x \sim z$. It follows that $R$ is an equivalence relation, and that $M^{\prime}$ is a complex manifold. It is clear that $M$ is a closed submanifold of $M^{\prime}$. This completes the proof of part (i) of Theorem 1.

Let us prove part (ii) of Theorem 1. Suppose we have two closed embeddings $\iota_{1}: M \rightarrow M_{1}^{\prime}$, $\iota_{2}: M \rightarrow M_{2}^{\prime}$ and an isomorphism $\phi: \iota_{1}^{-1} \mathcal{O}_{M_{1}^{\prime}} \cong \iota_{2}^{-1} \mathcal{O}_{M_{2}^{\prime}}$ of sheaves of $\mathbb{C}$-algebras commuting with natural surjections to $\mathcal{O}_{M}$. By Lemma 8, the isomorphism $\phi: \iota_{1}^{-1} \mathcal{O}_{M_{1}^{\prime}} \rightarrow \iota_{2}^{-1} \mathcal{O}_{M_{2}^{\prime}}$ is locally induced by a biholomorphic map which is the identity on $M$. Therefore we have a locally finite open covering $\left\{S_{i}: i \in I\right\}$ of $M$, open neighbourhoods $T_{i}$ of $S_{i}$ in $M_{2}^{\prime}$, and holomorphic maps $\varphi: T_{i} \rightarrow M_{1}^{\prime}$ such that $\varphi_{i}$ is the identity map on $T_{i} \cap M$ and $\left.\phi\right|_{S_{i}}=\varphi_{i}^{\star}$. Without loss of generality we may assume that $S_{i}$ is relatively compact in $M$. Choose an open covering $\left\{R_{i}: i \in I\right\}$ of $M$ such that $R_{i} \Subset S_{i}$, and choose an open tubular neighbourhood of $M$ in $M_{2}^{\prime}$. The tubular neighbourhood here is identified with a neighbourhood of the zero section of the normal bundle of $M$ in $M_{2}^{\prime}$; we choose a (fibrewise) Riemannian metric on it. We write $U(\epsilon) \subset M_{2}^{\prime}$ for the open tube of length $\epsilon>0$ over an open subset $U \subset M$. The maps $\varphi_{i}$ and $\varphi_{j}$ on the overlap $T_{i j}:=T_{i} \cap T_{j}$ coincide on an open neighbourhood 
$T_{i j}^{\circ} \subset T_{i j}$ of $S_{i j}:=S_{i} \cap S_{j}$. Since, for fixed $i \in I$, there are only finitely many $j \in I$ such that $S_{i j}$ is nonempty, there exists $\epsilon_{i}>0$ such that:

- $R_{i}\left(\epsilon_{i}\right) \subset T_{i}$

- $R_{i j}\left(\epsilon_{i}\right) \subset T_{i j}^{\circ}$ for all $j \in I$, where $R_{i j}:=R_{i} \cap R_{j} \Subset S_{i j}$.

Then the maps $\left\{\left.\varphi_{i}\right|_{R_{i}\left(\epsilon_{i}\right)}: i \in I\right\}$ coincide over each overlap $R_{i}\left(\epsilon_{i}\right) \cap R_{j}\left(\epsilon_{j}\right) \subset T_{i j}^{\circ}, i, j \in I$, and thus determine a global holomorphic map $\varphi$ on $N=\bigcup_{i \in I} R_{i}\left(\epsilon_{i}\right) \subset M_{2}^{\prime}$. The uniqueness of $\varphi$ as a germ is obvious. This completes the proof of Theorem 1.

\section{The Proof of Theorem 2}

Lemma 12. Let $\iota: M \rightarrow M^{\prime}$ be a closed embedding of complex manifolds, let $\mathcal{A}$ be the sheaf of $\mathbb{C}$-algebras $\mathcal{A}=\iota^{-1} \mathcal{O}_{M^{\prime}}$ over $M$, and let $\mathcal{B}$ be a locally finitely presented $\mathcal{A}=\iota^{-1} \mathcal{O}_{M^{\prime}}$-module. There exist:

- an open covering $\left\{V_{i}: i \in I\right\}$ of $M$ such that $V_{i}$ is relatively compact in $M$;

- for each $i \in I$, an open subset $W_{i}^{\prime}$ of $M^{\prime}$ such that $V_{i} \subset W_{i}^{\prime}$;

- for each $i, j \in I$, an open subset $A_{i j}$ of $M^{\prime}$ such that $V_{i j} \subset A_{i j} \subset W_{i j}^{\prime}$, where $V_{i j}:=V_{i} \cap V_{j}$ and $W_{i j}^{\prime}:=W_{i}^{\prime} \cap W_{j}^{\prime}$;

- for each $i, j, k \in I$, an open subset $B_{i j k}$ of $M^{\prime}$ such that $V_{i j k} \subset B_{i j k} \subset A_{i j k}$, where $V_{i j k}:=V_{i} \cap V_{j} \cap V_{k}$ and $A_{i j k}:=A_{i j} \cap A_{j k} \cap A_{i k}$;

- for each $i \in I$, a coherent $\mathcal{O}_{W_{i}^{\prime}}$-module $\mathcal{B}_{i}^{\prime}$ on $W_{i}^{\prime}$;

- for each $i \in I$, an isomorphism $\theta_{i}:\left.\iota^{-1} \mathcal{B}_{i}^{\prime} \cong \mathcal{B}\right|_{V_{i}}$ of $\mathcal{A}_{V_{i}}$-modules;

- for each $i, j \in I$, an isomorphism $\phi_{i j}:\left.\left.\mathcal{B}_{i}^{\prime}\right|_{A_{i j}} \cong \mathcal{B}_{j}^{\prime}\right|_{A_{i j}}$ of $\mathcal{O}_{A_{i j}}$-modules;

such that $A_{i j}, B_{i j k}$ are symmetric in their indices and that the diagrams:
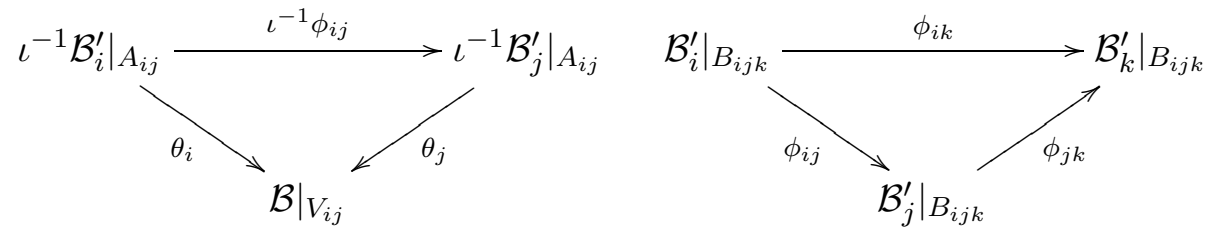

commute for each $i, j, k \in I$.

Proof of Lemma 12. We take open coverings $\left\{V_{i}: i \in I\right\},\left\{W_{i}: i \in I\right\}$ of $M$ by Stein open subsets $V_{i}, W_{i}$ such that $V_{i} \Subset W_{i} \Subset M$ and that $\left.\mathcal{B}\right|_{W_{i}}$ has a finite presentation

$$
\left.\mathcal{A}_{W_{i}}^{\oplus k_{i}} \stackrel{\xi_{i}}{\longrightarrow} \mathcal{A}_{W_{i}}^{\oplus l_{i}} \stackrel{\eta_{i}}{\longrightarrow} \mathcal{B}\right|_{W_{i}} \longrightarrow 0
$$

as in (2). For example, we can take $V_{i}, W_{i}$ to be small open balls centered at the same point of a co-ordinate chart. The $\mathcal{A}$-module homomorphism $\xi_{i}: \mathcal{A}_{W_{i}}^{\oplus k_{i}} \rightarrow \mathcal{A}_{W_{i}}^{\oplus l_{i}}$ extends to an $\mathcal{O}$-module homomorphism $\xi_{i}^{\prime}: \mathcal{O}_{W_{i}^{\prime}}^{\oplus k_{i}} \rightarrow \mathcal{O}_{W_{i}^{\prime}}^{\oplus l_{i}}$ on a neighbourhood $W_{i}^{\prime}$ of $W_{i}$ in $M^{\prime}$ and defines a coherent $\mathcal{O}_{W_{i}^{\prime}}$-module $\mathcal{B}_{i}^{\prime}$ by the exact sequence:

$$
\mathcal{O}_{W_{i}^{\prime}}^{\oplus k_{i}} \stackrel{\xi_{i}^{\prime}}{\longrightarrow} \mathcal{O}_{W_{i}^{\prime}}^{\oplus l_{i}} \stackrel{\eta_{i}^{\prime}}{\longrightarrow} \mathcal{B}_{i}^{\prime} \longrightarrow 0
$$

By construction there is an $\mathcal{A}$-module isomorphism $\theta_{i}:\left.\iota^{-1} \mathcal{B}_{i}^{\prime} \rightarrow \mathcal{B}\right|_{W_{i}}$. For each pair $(i, j)$ such that $V_{i j}:=V_{i} \cap V_{j}$ is nonempty, we construct a homomorphism $\phi_{i j}$ from $\mathcal{B}_{i}^{\prime}$ to $\mathcal{B}_{j}^{\prime}$. Let $e_{1}, \ldots, e_{l_{i}}$ denote the standard basis of $\mathcal{O}_{W_{i}}^{\oplus l_{i}}$. For each $1 \leq a \leq l_{i}$, the image $\eta_{i}\left(e_{a}\right)$ is a section of $\mathcal{B}_{i}^{\prime}$ and induces a section $s_{a}$ of $\left.\iota^{-1} \mathcal{B}_{i}^{\prime} \cong \mathcal{B}\right|_{W_{i}}$. Via the isomorphism $\left.\left.\iota^{-1} \mathcal{B}_{j}^{\prime}\right|_{W_{i j}} \cong \mathcal{B}\right|_{W_{i j}}$, the restriction $\left.s_{a}\right|_{W_{i j}}$ can be lifted to a section $t_{a}$ of $\mathcal{B}_{j}^{\prime}$ over an open neighbourhood $C_{i j}$ of $W_{i j}$ in $W_{i j}^{\prime}$. Because the intersection $V_{i j}$ of Stein open sets $V_{i}, V_{j}$ is Stein and because $V_{i j} \Subset W_{i j}$, we can find a Stein 
open neighbourhood $A_{i j}$ of $V_{i j}$ in $C_{i j}$. Because $A_{i j}$ is Stein, we can find a lift $u_{a} \in \Gamma\left(A_{i j}, \mathcal{O}^{\oplus l_{j}}\right)$ of $\left.t_{a}\right|_{A_{i j}}$ such that $\eta_{j}\left(u_{a}\right)=\left.t_{a}\right|_{A_{i j}}$. The sections $u_{1}, \ldots, u_{l_{i}}$ define a homomorphism $\psi_{i j}: \mathcal{O}_{A_{i j}}^{\oplus l_{i}} \rightarrow \mathcal{O}_{A_{i j}}^{\oplus l_{j}}$ sending $e_{a}$ to $u_{a}$.

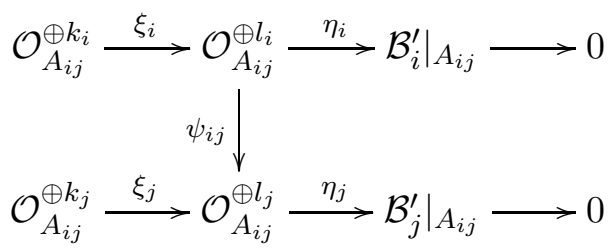

We claim that, after shrinking $A_{i j}$ if necessary, $\psi_{i j}$ induces a homomorphism $\phi_{i j}:\left.\left.\mathcal{B}_{i}^{\prime}\right|_{A_{i j}} \rightarrow \mathcal{B}_{j}^{\prime}\right|_{A_{i j}}$. It suffices to show that the composition $\eta_{j} \circ \psi_{i j} \circ \xi_{i}$ is zero in a neighbourhood of $V_{i j}$. By construction, $\eta_{i}(v)$ and $\eta_{j} \circ \psi_{i j}(v)$ define the same section of $\mathcal{B}$ over $V_{i j}$ for every $v \in \Gamma\left(A_{i j}, \mathcal{O}_{A_{i j}}^{\oplus l_{i}}\right)$. Hence for $w \in \Gamma\left(A_{i j}, \mathcal{O}_{A_{i j}}^{\oplus k_{i}}\right), \eta_{j} \circ \psi_{i j} \circ \xi_{i}(w)$ and $\eta_{i} \circ \xi_{i}(w)=0$ define the same section of $\mathcal{B}$ over $V_{i j}$. This means that $\eta_{j} \circ \psi_{i j} \circ \xi_{i}(w)$ vanishes in a neighbourhood of $V_{i j}$, and the claim follows. It is clear that the first diagram in (4) commutes. We can also assume that $A_{i j}=A_{j i}$ by replacing $A_{i j}$ with $A_{i j} \cap A_{j i}$ and restricting $\phi_{i j}$ to it if necessary.

Finally we find an open subset $B_{i j k}$ of $A_{i j k}=A_{i j} \cap A_{j k} \cap A_{i j}$ containing $V_{i j k}=V_{i} \cap V_{j} \cap V_{k}$ on which the second diagram in (4) commutes (i.e. the cocycle condition holds). But this is straightforward, because $\phi_{j k} \circ \phi_{i j}=\phi_{i k}$ holds at the stalk of each $x \in V_{i j k}$.

Let $\iota: M \hookrightarrow M^{\prime}, \mathcal{A}$, and $\mathcal{B}$ be as in the statement of Theorem 2. Take the data constructed in Lemma 12. By taking a refinement if necessary, we may assume that the open covering $\left\{V_{i}: i \in I\right\}$ is locally finite. Choose an open covering $\left\{U_{i}: i \in I\right\}$ of $M$ such that $U_{i} \Subset V_{i}$. Take an open tubular neighbourhood of $M$ in $M^{\prime}$ and, as in the proof of Theorem 1, fix a fibrewise Riemannian metric on it. For a open subset $U$ of $M$ and $\epsilon>0$, we denote by $U(\epsilon) \subset M^{\prime}$ the open tube of length $\epsilon$ over $U$. For each $i \in I$, there are only finitely many $j \in I$ such that the intersection $V_{i j}$ is non-empty. Therefore we can find $\epsilon_{i}>0$ such that:

- $U_{i}\left(\epsilon_{i}\right) \subset W_{i}^{\prime}$;

- $U_{i j}\left(\epsilon_{i}\right) \subset A_{i j}$ for all $j \in I$, where $U_{i j}:=U_{i} \cap U_{j}$;

- $U_{i j k}\left(\epsilon_{i}\right) \subset B_{i j k}$ for all $j, k \in I$, where $U_{i j k}:=U_{i} \cap U_{j} \cap U_{k}$.

Then the coherent sheaves $\left.\mathcal{B}_{i}^{\prime}\right|_{U_{i}\left(\epsilon_{i}\right)}$ glue together via the homomorphisms $\left.\phi_{i j}\right|_{U_{i}\left(\epsilon_{i}\right) \cap U_{j}\left(\epsilon_{j}\right)}$ to give a global coherent $\mathcal{O}_{N}$-module $\mathcal{B}^{\prime}$ on $N=\bigcup_{i \in I} U_{i}\left(\epsilon_{i}\right)$. It is clear that there is an isomorphism $\iota^{-1} \mathcal{B}^{\prime} \cong \mathcal{B}$ of $\mathcal{A}$-modules. This completes the proof of part (i) of Theorem 2 .

Let us prove part (ii) of Theorem 2. Suppose that we have coherent $\mathcal{O}_{N}$-modules $\mathcal{B}_{1}^{\prime}$ and $\mathcal{B}_{2}^{\prime}$ and a isomorphisms $\iota^{-1} \mathcal{B}_{1}^{\prime} \cong \mathcal{B} \cong \iota^{-1} \mathcal{B}_{2}^{\prime}$ of $\mathcal{A}$-modules. Let $\phi: \iota^{-1} \mathcal{B}_{1}^{\prime} \rightarrow \iota^{-1} \mathcal{B}_{2}^{\prime}$ denote the composite isomorphism. We can find a locally finite open covering $\left\{S_{i}: i \in I\right\}$ of $M$ together with a family $\left\{T_{i}: i \in I\right\}$ of open subsets of $M^{\prime}$ such that $S_{i} \Subset T_{i}$ and $\left.\mathcal{B}_{1}^{\prime}\right|_{T_{i}}$ and $\left.\mathcal{B}_{2}^{\prime}\right|_{T_{i}}$ have finite presentations as $\mathcal{O}_{T_{i}}$-modules. Without loss of generality we may assume that $S_{i}$ is Stein. The argument in the proof of Lemma 12 shows that we can find an open neighbourhood $T_{i}^{\circ}$ of $S_{i}$ in $T_{i}$ and a homomorphism $\Phi_{i}:\left.\left.\mathcal{B}_{1}^{\prime}\right|_{T_{i}^{\circ}} \rightarrow \mathcal{B}_{2}^{\prime}\right|_{T_{i}^{\circ}}$ such that $\iota^{-1} \Phi_{i}=\left.\phi\right|_{S_{i}}$. For each $i, j \in I$, there exists an open subset $P_{i j}$ of $T_{i}^{\circ} \cap T_{j}^{\circ}$ containing $S_{i j}:=S_{i} \cap S_{j}$ such that $\left.\Phi_{i}\right|_{P_{i j}}=\left.\Phi_{j}\right|_{P_{i j}}$. Take an open covering $\left\{R_{i}: i \in I\right\}$ of $M$ such that $R_{i} \Subset S_{i}$. As before, we choose a tubular neighbourhood of $M$ in $M^{\prime}$ and a (fibrewise) Riemannian metric on it, denoting by $U(\epsilon)$ the open $\epsilon$-tube over the open subset $U \subset M$. Then for each $i \in I$, there exists $\epsilon_{i}>0$ such that:

- $R_{i}\left(\epsilon_{i}\right) \subset T_{i}^{\circ}$

- $R_{i j}\left(\epsilon_{i}\right) \subset P_{i j}$

because there are only finitely many $j \in I$ such that $S_{i j}$ is nonempty. Now the homomorphisms $\left.\Phi_{i}\right|_{R_{i}\left(\epsilon_{i}\right)}$ glue together to define a global homomorphism $\Phi:\left.\left.\mathcal{B}_{1}^{\prime}\right|_{P} \rightarrow \mathcal{B}_{2}^{\prime}\right|_{P}$ over $P=\bigcup_{i \in I} R_{i}\left(\epsilon_{i}\right)$ such that $\phi=\iota^{-1} \Phi$. The uniqueness of $\Phi$ as a germ is obvious. This completes the proof of Theorem 2 . 


\section{Global Unfolding of TeP Structures}

As an application of our results we now prove a global unfolding theorem for TEP structures, by globalizing the reconstruction theorem for germs of TEP structures due to Hertling-Manin [7]. This global unfolding theorem has applications in mirror symmetry and the study of quantum cohomology $[1, \S 8.1 .6]$. TEP structures were introduced by Hertling [6]; they are closely related to Dubrovin's notion of Frobenius manifold [3,4,7].

Definition 13 (TEP structure). Let $M$ be a complex manifold. A TEP structure $\left(\mathcal{F}, \nabla,(\cdot, \cdot)_{\mathcal{F}}\right)$

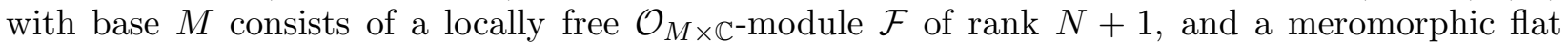
connection

$$
\nabla: \mathcal{F} \rightarrow\left(\pi^{*} \Omega_{M}^{1} \oplus \mathcal{O}_{M \times \mathbb{C}} z^{-1} d z\right) \otimes_{\mathcal{O}_{M \times \mathbb{C}}} \mathcal{F}(M \times\{0\})
$$

so that for $f \in \mathcal{O}_{M \times \mathbb{C}}, s \in \mathcal{F}$, and tangent vector fields $v_{1}, v_{2} \in \Theta_{M \times \mathbb{C}}$ :

$$
\left.\nabla(f s)=d f \otimes s+f \nabla s \quad\left[\nabla_{v_{1}}, \nabla_{v_{2}}\right]=\nabla_{\left[v_{1}, v_{2}\right.}\right]
$$

together with a non-degenerate pairing

$$
(\cdot, \cdot)_{\mathcal{F}}:(-)^{*} \mathcal{F} \otimes_{\mathcal{O}_{M \times \mathbb{C}}} \mathcal{F} \rightarrow \mathcal{O}_{M \times \mathbb{C}}
$$

which satisfies

$$
\begin{aligned}
\left((-)^{*} s_{1}, s_{2}\right)_{\mathcal{F}} & =(-)^{*}\left((-)^{*} s_{2}, s_{1}\right)_{\mathcal{F}} \\
d\left((-)^{*} s_{1}, s_{2}\right)_{\mathcal{F}} & =\left((-)^{*} \nabla s_{1}, s_{2}\right)_{\mathcal{F}}+\left((-)^{*} s_{1}, \nabla s_{2}\right)_{\mathcal{F}}
\end{aligned}
$$

for $s_{1}, s_{2} \in \mathcal{F}$. Here $\mathcal{F}(M \times\{0\})$ denotes the sheaf of sections of $\mathcal{F}$ with poles of order at most 1 along the divisor $M \times\{0\} \subset M \times \mathbb{C}$.

Definition 14 (Miniversality). Let $M$ be a complex manifold. A TEP structure $\left(\mathcal{F}, \nabla,(\cdot, \cdot)_{\mathcal{F}}\right)$ with base $M$ is called miniversal if for each $y \in M$, the set

$$
\left\{\left.x \in \mathcal{F}\right|_{(y, 0)} \text { : the map }\left.T_{y} M \rightarrow \mathcal{F}\right|_{(y, 0)},\left.v \mapsto\left(z \nabla_{v} x\right)\right|_{(y, 0)} \text { is an isomorphism }\right\}
$$

is non-empty in the fiber $\left.\mathcal{F}\right|_{(y, 0)}$.

Theorem 15 (Global unfolding for TEP structures). Let $M$ be a complex manifold and $\left(\mathcal{F}, \nabla,(\cdot, \cdot)_{\mathcal{F}}\right)$ a TEP structure with base $M$. Suppose that for each $y \in M$, there exists a section $\zeta$ of $\mathcal{F}$ over a neighbourhood of $(y, 0) \in M \times \mathbb{C}$ such that:

(IC): the map $\left.T_{y} M \rightarrow \mathcal{F}\right|_{(y, 0)}$ defined by $\left.v \mapsto z \nabla_{v} \zeta\right|_{(y, 0)}$ is injective;

(GC): the fiber $\left.\mathcal{F}\right|_{(y, 0)}$ is generated by iterated derivatives

$$
\left.\left(z^{2} \nabla_{\partial_{z}}\right)^{l} z \nabla_{v_{1}} \cdots z \nabla_{v_{k}} \zeta\right|_{(y, 0)} \quad l \geq 0
$$

with respect to local vector fields $v_{1}, \ldots, v_{k}$ on $M$ near $y$ and $z^{2} \partial_{z}$.

Then there exist a complex manifold $M^{\prime}$, a miniversal TEP structure $\left(\mathcal{F}^{\prime}, \nabla^{\prime},(\cdot, \cdot)_{\mathcal{F}^{\prime}}\right)$ with base $M^{\prime}$, and a closed embedding $\iota: M \rightarrow M^{\prime}$ such that:

$$
\iota^{\star}\left(\mathcal{F}^{\prime}, \nabla^{\prime},(\cdot, \cdot)_{\mathcal{F}^{\prime}}\right)=\left(\mathcal{F}, \nabla,(\cdot, \cdot)_{\mathcal{F}}\right)
$$

Furthermore the manifold-germ $M^{\prime}$ and the TEP structure $\left(\mathcal{F}^{\prime}, \nabla^{\prime},(\cdot, \cdot)_{\mathcal{F}^{\prime}}\right)$ are unique up to unique isomorphism in the sense of Theorem 1 and 2.

Proof. Combine Theorems 1 and 2 with the universal unfolding theorem for germs of TEP structures proved by Hertling-Manin [7, Theorem 2.5, Lemma 3.2].

Analogous global unfolding theorems for TE structures [7], log-trTLEP structures [10], and so on can be proved in exactly the same way. Global unfoldings of log-trTLEP structures have interesting applications in Gromov-Witten theory [2]. 


\section{REFERENCES}

1. Tom Coates and Hiroshi Iritani. A Fock sheaf for Givental quantization. in preparation.

2. Tom Coates and Hiroshi Iritani. Modularity of Gromov-Witten potentials for local $\mathbb{P}^{2}$. In preparation.

3. Boris Dubrovin. Geometry of 2D topological field theories. In Integrable systems and quantum groups (Montecatini Terme, 1993), volume 1620 of Lecture Notes in Math., pages 120-348. Springer, Berlin, 1996.

4. Boris Dubrovin. Geometry and analytic theory of Frobenius manifolds. In Proceedings of the International Congress of Mathematicians, Vol. II (Berlin, 1998), number Extra Vol. II, pages 315-326, 1998.

5. Kenji Fukaya, Yong-Geun Oh, Hiroshi Ohta, and Kaoru Ono. Technical details on Kuranishi structure and virtual fundamental chain. arXiv:1209.4410 [math.SG], 2012.

6. Claus Hertling. $t t^{*}$ geometry, Frobenius manifolds, their connections, and the construction for singularities. $J$. Reine Angew. Math., 555:77-161, 2003.

7. Claus Hertling and Yuri Manin. Unfoldings of meromorphic connections and a construction of Frobenius manifolds. In Frobenius manifolds, Aspects Math., E36, pages 113-144. Vieweg, Wiesbaden, 2004.

8. Dominic Joyce. D-manifolds and d-orbifolds: a theory of derived differential geometry. Available at http://people.maths.ox.ac.uk/joyce/dmbook.pdf, 2012.

9. Masaki Kashiwara. D-modules and microlocal calculus, volume 217 of Translations of Mathematical Monographs. American Mathematical Society, Providence, RI, 2003. Translated from the 2000 Japanese original by Mutsumi Saito, Iwanami Series in Modern Mathematics.

10. Thomas Reichelt. A construction of Frobenius manifolds with logarithmic poles and applications. Comm. Math. Phys., 287(3):1145-1187, 2009.

11. Masahiro Shiota. Some results on formal power series and differentiable functions. Publ. Res. Inst. Math. Sci., 12(1):49-53, 1967/77.

12. H. Whitney and F. Bruhat. Quelques propriétés fondamentales des ensembles analytiques-réels. Comment. Math. Helv., 33:132-160, 1959.

E-mail address: t.coates@imperial.ac.uk

E-mail address: iritani@math.kyoto-u.ac.jp 